7th International Symposium on Superalloy 718 and Derivatives Edited by: E.A. Ott, J.R. Groh, A. Banik, I. Dempster, T.P. Gabb, R. Helmink, X. Liu, A. Mitchell, G.P. Sjöberg, and A. Wusatowska-Sarnek TMS (The Minerals, Metals \& Materials Society), 2010

\title{
Characterization of microstructures containing abnormal grain growth zones in Alloy 718
}

\author{
Benjamin Flageolet $^{1}$, O. Yousfi ${ }^{1,2}$, Y. Dahan ${ }^{1}$, P. Villechaise ${ }^{3}$, J. Cormier $^{3}$ \\ ${ }^{1}$ Aubert \& Duval Pamiers, 75 Bd de la Libération, 09100 Pamiers, France \\ ${ }^{2}$ Simap, Grenoble INP, Domaine Universitaire BP75, Saint Martin d'Hères cedex, France \\ ${ }^{3}$ Institut Pprime, CNRS - ENSMA - Université de Poitiers, UPR CNRS 3346, Département de \\ Physique et Mécanique des Matériaux, 1 Avenue Clément Ader, BP40109, 86961 Futuroscope \\ Chasseneuil, France
}

Keywords: 718 alloy, fatigue, crack initiation, abnormal grain growth

\begin{abstract}
A well known issue encountered by forgers transforming Alloy 718 consists in the appearance of coarse grain zones during the thermo-mechanical process. Although some industrial solutions have been implemented in order to get rid of this type of microstructural defect, the consequences of the presence of such coarse grained areas on the material's mechanical properties are of great interest.

In this work, some zones that have undergone abnormal grain growth (AGG) have primarily been characterized from a microstructural point of view by means of both optical and EBSD observations. Special attention has been paid to grain orientation, grain boundaries and grain size distributions. In addition the effects of these microstructures on $350^{\circ} \mathrm{C}$ deformation controlled fatigue crack initiation mechanisms and the subsequent lifetime have been assessed as well as on $350^{\circ} \mathrm{C}$ tensile properties have also been evaluated.

The results will be discussed with regard to microstructure/mechanical behavior relationships.

\section{Introduction}

Modern jet engines developments imply a constant improvement of the properties of the materials that are used, especially for all rotating parts. The Ni-Fe based superalloy 718 has been widely used for decades for turbine disk applications, requiring very high mechanical properties at temperatures as high as $650^{\circ} \mathrm{C}$. The properties exhibited by this alloy are strongly dependent on the thermomechanical processing during the manufacturing of the disks through the microstructure obtained. Among all microstructural parameters required by engine manufacturers, the grain size is the most important parameter where an ideal creep-fatigue compromise corresponds to an average grain size of about $15 \mu \mathrm{m}$ [12].

In this light, all Alloy 718 forgers manufacturing jet engine disks, design their forging processes in order to achieve a very good recrystallization rate and a fine grain microstructure. In spite of the very wide use and experience accumulated on this alloy, and due to the complexity of the forging processes, the tendency to reduce the input weight and to the complexity of the shapes to be forged, uncontrolled grain growth phenomena may still occur in some instances.

Abnormal grain growth phenomena have been the study object of many papers, either focusing on a particular case associated to an alloy, or on general theory. It is interesting to note that two main approaches can be distinguished in the existing hypothesis. The first one considers the materials texture prior to heating, thus explaining local differences in the grain boundaries
\end{abstract}


mobility [1, 2, 3]. The second one essentially takes the initial grain size distribution before heating operations into consideration, with or without taking the presence of a second phase into account $[4,5,6,7]$.

Although some efficient solutions can be found to avoid the formation of AGG zones during the forging processes, there are, to our knowledge, very few data available in the literature on the characterization of such microstructures, neither from a metallographic nor from a mechanical properties point of view. The work presented in this paper consequently aims at characterizing and comparing the microstructure of a forging in the coarse grained zones with the "clean" zones. The impact of the presence of coarse grain zones on tensile and low cycle fatigue properties at $350^{\circ} \mathrm{C}$ has also been assessed.

\section{Material and experimental}

The material used in this study consists of an axial/radial slice cut from a forged and heat treated (direct aged) 718 turbine disk. The slice was first polished and etched using an oxalic acid solution and examined by optical microscopy. Based on this preliminary observation, two different zones were found corresponding to an increase of the coarse grain content. The first zone (denoted as "zone 1") corresponds to a "clean" area exhibiting only normal grain growth, while zone 2 contains AGG. Figure 1 shows typical images corresponding to each of these two zones chosen for characterization, as well as their position in the forging.

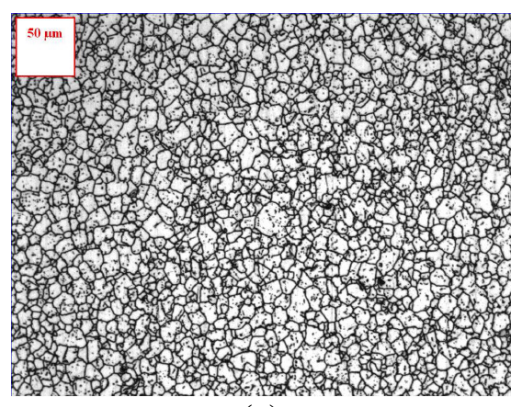

(a)

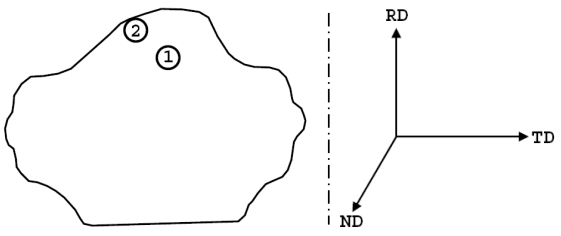

(b)

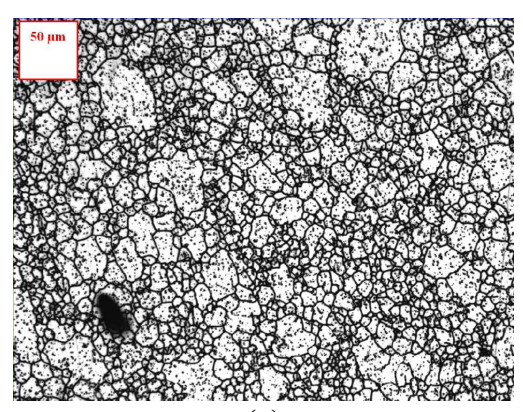

(c)

Figure 1: Zones chosen for EBSD characterization. (a) zone1, (C) zone 2

These two zones have been chosen in order to be able to characterize what is assumed to correspond to intermediate states of the microstructure during abnormal grain growth. Indeed the microstructure can coarsen in such an extent that in some cases, the size of the coarsest grains can reach 70 to $100 \mu \mathrm{m}$, corresponding to about ASTM 5. Figure 2 shows the worst microstructure that can be observed following AGG phenomenon. This microstructure consists of coarse grains, larger than $70 \mu \mathrm{m}$, in a fine grained ASTM $10(15 \mu \mathrm{m})$ matrix. It is also interesting to notice in Figure 2 that the coarse grains contain a significant amount of delta phase, which is basically used to pin the grain boundaries and hinder their migration. In addition to this, the grain boundaries of the coarse grain do not have the same appearance as in the fine grain area. This is observed for various etching solutions. Grain boundaries are indeed very round shaped and can not always clearly be identified.

Two small samples were then cut within the two characteristic areas and characterized using EBSD technique after a suitable preparation sequence including mechanical polishing up to a $1 \mu \mathrm{m}$ grade and a subsequent long colloidal silica polishing. A JEOL 7000F SEM-FEG equipped with an EBSD device / OIM ${ }^{\circledR}$ software were used for those analyzes. The specimens were characterized using a $0.5 \mu \mathrm{m}$ scanning step. $1 \mathrm{~mm} \times 0.5 \mathrm{~mm}$ and $1.5 \times 0.5 \mathrm{~mm}$ scanning areas were 
considered for zones 1 and 2 respectively. For each specimen, the grain size distribution and reconstructed grain size map have been extracted based on a minimum grain boundary misorientation criterion of 15 degrees and by taking twin boundaries ( $\Sigma 3$ ) into account. Crystallographic textures were analyzed using pole figures.

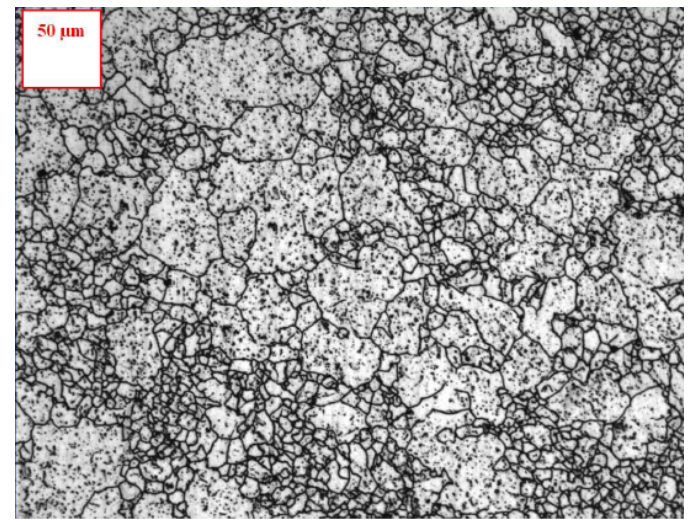

Figure 2: Worst microstructure observed after abnormal grain growth

In addition to EBSD examinations, 21 small dimension cylindrical tensile specimens (3.5mm in diameter and $16 \mathrm{~mm}$ in gage length) as well as 12 cylindrical low cycle fatigue specimens $(4.37 \mathrm{~mm}$ in diameter and $13 \mathrm{~mm}$ in gage length) have been cut from specific locations of the forging where AGG microstructures were expected to be obtained. Tensile specimens were tested at $350^{\circ} \mathrm{C}$ under controlled strain rate mode, according to ASTM E8. Fatigue specimens have been tested at $350^{\circ} \mathrm{C}$ according to ASTM E604 in total strain control mode with a sinusoidal waveform, under a strain ratio $\mathrm{R} \varepsilon=0$ and a frequency of $1 \mathrm{~Hz}$. The maximum total strain level has been chosen to be $0.56 \%$. The results obtained on samples suspected to contain AGG have been compared with results obtained in the same conditions on the standard material (i.e. free of $\mathrm{AGG}$ ).

\section{Results}

\section{$\underline{\text { Microstructural Results }}$}

Figure 3a shows the as reconstructed grain microstructure obtained in zone 1 which does not contain any identified AGG zone. The red lines correspond to $\Sigma 3$ [111] twin boundaries. An interesting point is that in comparison to the initial optical observation, the material contains a significant amount of isolated grains that have a grain size larger than the average grain size of the whole population. It is thus not as homogeneous as it could have been imagined based on optical observations. The grain size distribution obtained on this specimen (see Figure 3c) exhibits a very small average grain size, close to $10 \mu \mathrm{m}$. Additionally, a significant part of the population is bellow $9 \mu \mathrm{m}$. Occasional grains between 25 and $40 \mu \mathrm{m}$ can also be identified. The microstructure is consequently much more heterogeneous than what was expected based on the initial optical observations.

The pole figures obtained from the EBSD data are presented in Figure 3c. Although giving a very weak maximum texture index (1.8), the specimen tends to exhibit a very slight deformation texture. 


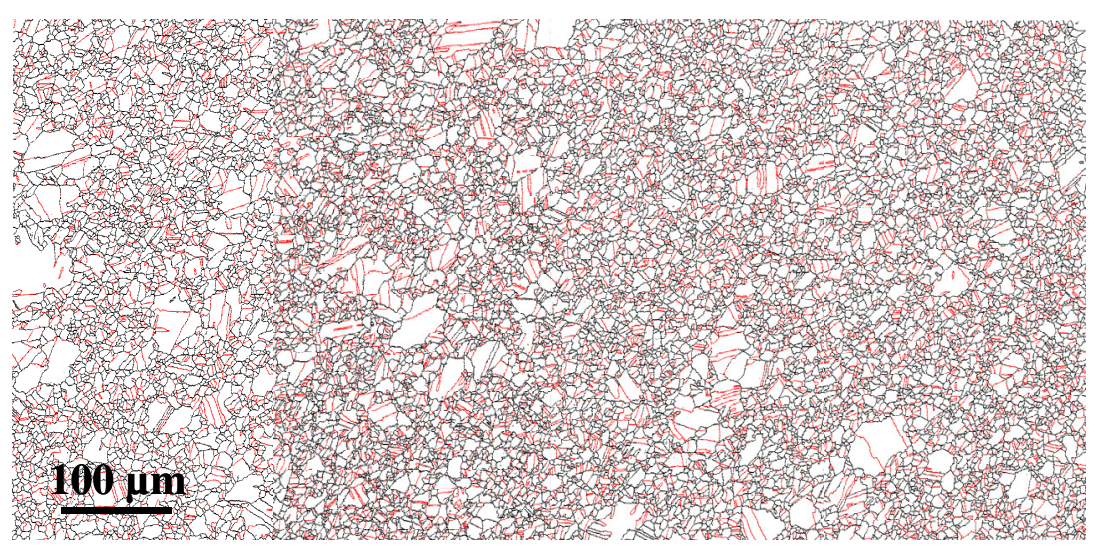

(a)

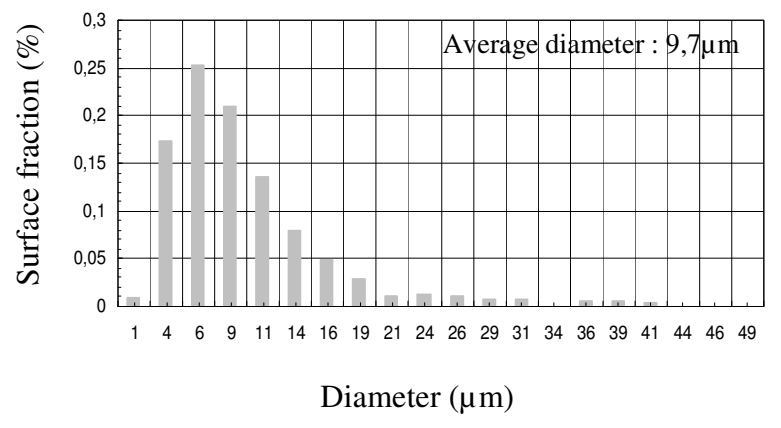

(b)
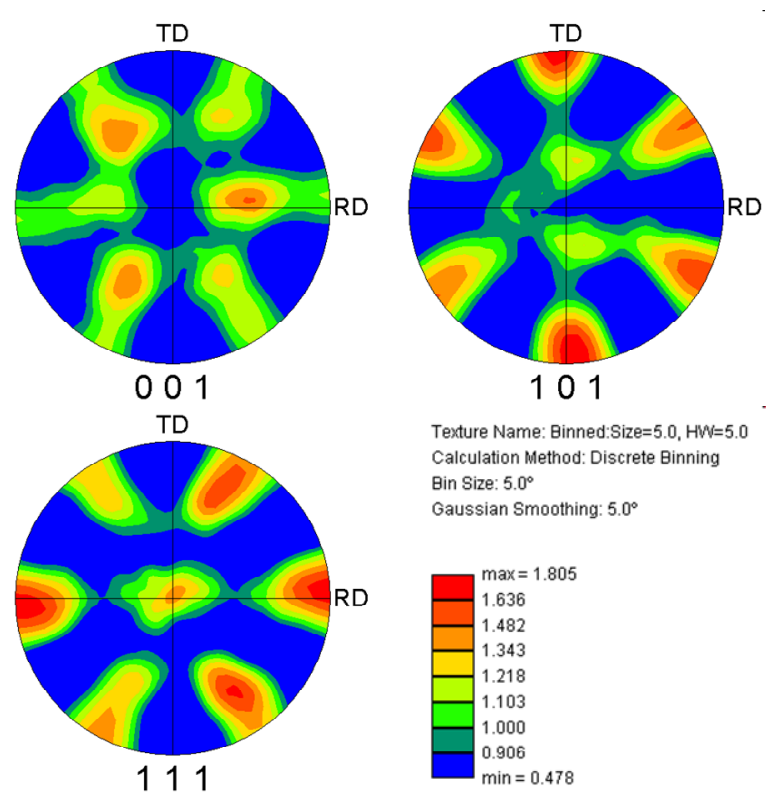

Texture Name: Binned:Size $=5.0, \mathrm{HW}=5.0$ Calculation Method: Discrete Binning Bin Size: $5.0^{\circ}$ Gaussian Smoothing: $5.0^{\circ}$

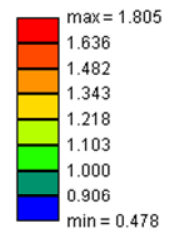

(c)

Figure 3 : Zone 1 EBSD results. (a) Reconstructed grain microstructure. $\Sigma 3$ [111] twin boundaries appear in red. (b) Grain size distribution. (c) Pole figures

Figure 4 gathers the same results as described above for AGG containing zones (zone 2 in the specimen). The as reconstructed grain map is very similar to the one obtained in zone 1 and includes some coarse grains up to about $50 \mu \mathrm{m}$. The grain size is also almost the same as for zone 1 , with a $10.9 \mu \mathrm{m}$ average value. The grain size distribution consequently does not change much between sound and AGG zone. Only some very large grains, often containing many twin boundaries appear in the AGG zone.

The pole figures obtained for the specimen containing AGG (Fig. 4) do not exhibit the same texture as for specimen 1 (Figure 3). In both cases however, the maximum texture index, is very low. Texture in the area where abnormal grain growth is observed is consequently very close to a random texture.

Some comparative analyzes were also performed to assess more precisely the possible differences between zone 1 and zone 2 in terms of special boundaries, low angle (i.e. below 15 degrees) boundaries and high angle boundaries linear fraction. The results (see Table 1) reveal that the linear fractions of high angle boundaries and twin boundaries are significantly higher in 
zone 2 while the fraction of low angle boundaries is lower. This tends to show that the AGG containing zone has undergone a recovery process. Consequently, the average mobility of the grain boundaries in zone 2 specimen should be higher than the average mobility of the grain boundaries of zone 1 specimen. The material in zone 2 is thus in a more favorable configuration to undergo further grain coarsening. The higher amount of annealing twin boundaries in the AGG containing specimen could be a direct consequence of a higher area fraction of coarse grains.

Table 1: Grain boundary linear fraction measurements on zone 1 and zone 2 specimens

\begin{tabular}{|c|c|c|c|}
\hline Specimen & $\mathbf{\Sigma 3}(\boldsymbol{\%})$ & LAB (\%) & HAB (\%) \\
\hline Zone 1 (no AGG) & 11 & 57 & 43 \\
\hline Zone 2 & 23 & 24 & 76 \\
\hline
\end{tabular}

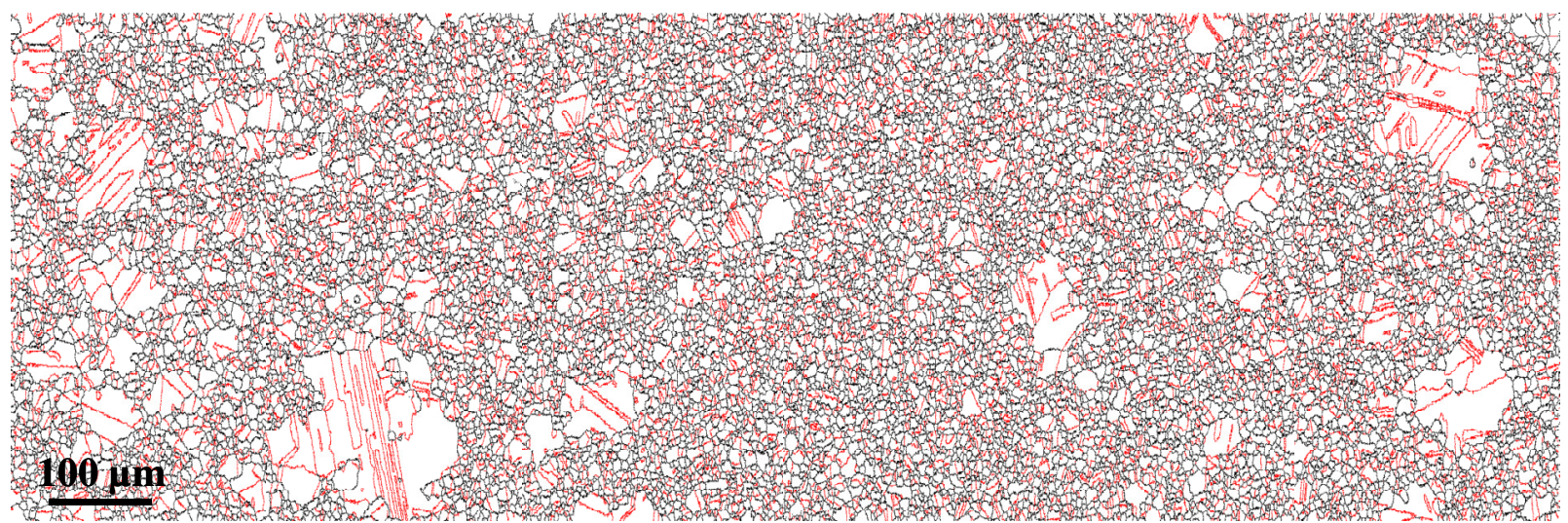

(a)

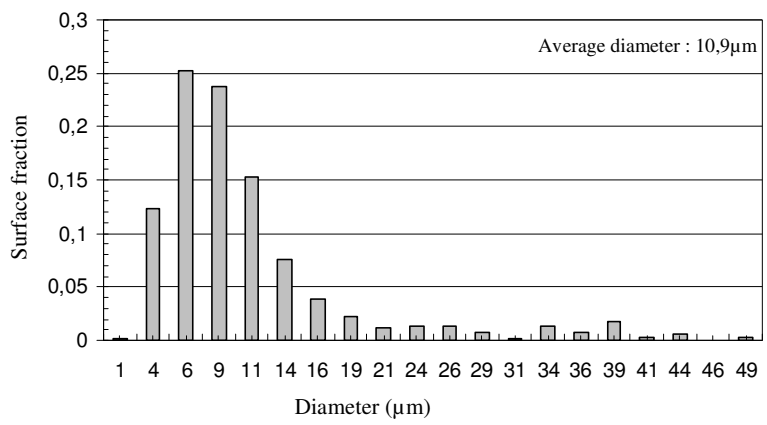

(b)

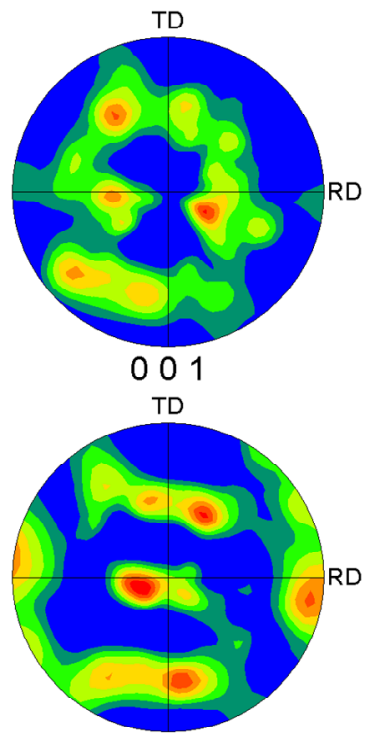

111

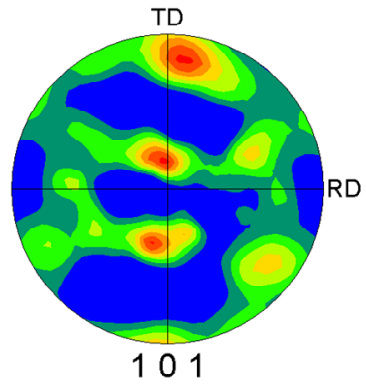

Texture Name: Binned:Size $=5.0, \mathrm{HW}=5.0$ Calculation Method: Discrete Binning Bin Size: $5.0^{\circ}$ Gaussian Smoothing: $5.0^{\circ}$

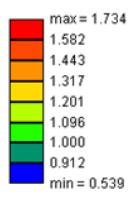

(c)

Figure 4: Zone 2 EBSD results. (a) Reconstructed grain microstructure. $\Sigma 3$ [111] twin boundaries appear in red. (b) Grain size distribution. (c) Pole figures

Based on these observations, the abnormal growth we observe does not seem to be promoted by a locally highly textured zone in a specific zone of the forging. Nevertheless, these results do not allow us at that time to eliminate the hypothesis that the abnormal grain growth could be related to crystallographic effects. Some additional assessments of the local grain boundary orientation 
of the coarse grains in comparison with the average grain boundary orientation in a fine grained area are subjects for future studies.

\section{$\underline{\text { Mechanical Tests Results }}$}

As previously mentioned, 21 tensile specimens were cut in some areas of a disk identified as AGG containing. Those specimens were tested at $350^{\circ} \mathrm{C}$ and the results compared to the results obtained from material free from coarse grain areas. The results are presented in Figure 5 using a box and whiskers representation.

It appears that the results obtained on the specimens cut from AGG zones are very similar to the ones obtained on the part without AGG. Only the yield stress (YS) seems to be significantly different in the two sets of data, with about $20 \mathrm{MPa}$ less in the case of the AGG containing specimens. Statistical T tests performed to compare the two sets of data confirm that, with a 95\% confidence, there are two YS populations while there is only one for UTS, elongation and reduction of area.

All the specimens have been examined longitudinally by means of optical microscopy in order to ensure that they contain coarse grain areas. The result is that none of the tensile specimens clearly contains coarse grain areas from the cross sections examined. It is consequently not possible to confirm that localized coarse grain areas have a significant impact on the tensile properties of the alloy. Because of the small size of the specimens we may not have succeed to cut the tensile samples within coarse grain zones. However, as far as coarse grain areas are much localized, their presence in the specimens may not have any significant consequences on the macroscopic tensile curves. On the other hand, islands of coarse grains contained in a fine grained matrix may have important effects on the fatigue behavior of the material. This is the reason why LCF tests have also been performed in order to assess these potential effects.

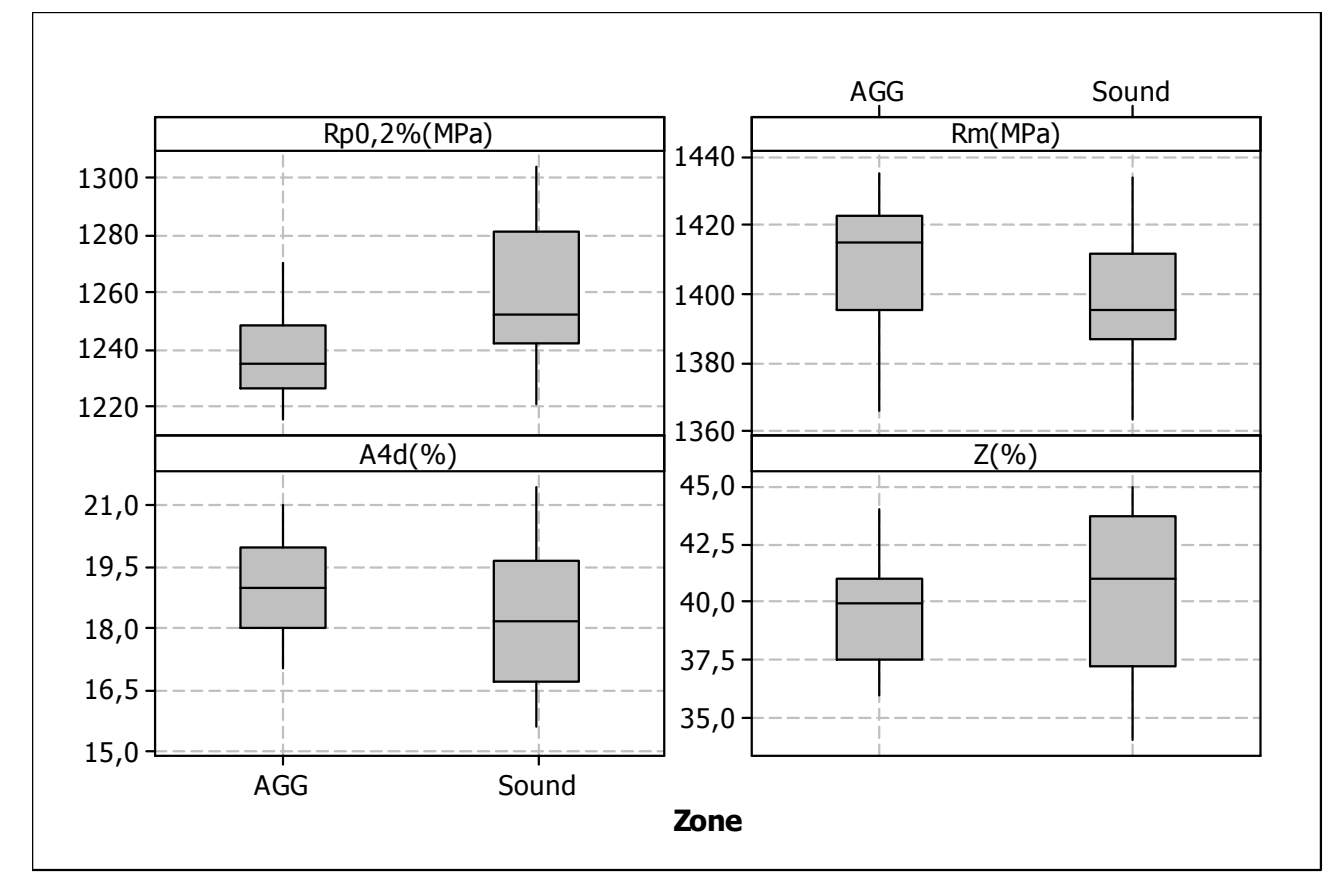

Figure 5: $350^{\circ} \mathrm{C}$ Tensile test results. Comparison with a part free from AGG zones 
The results obtained on the fatigue specimens are presented in Fig. 6 and compared to the results obtained on a part which does not contain coarse grain areas. Contrary to tensile tests, two different populations are clearly obtained, with a strong decrease of the number of cycles to failure when the specimens are machined in AGG zones. The lifetime drop can indeed reach one order of magnitude and is associated with an increased scatter.

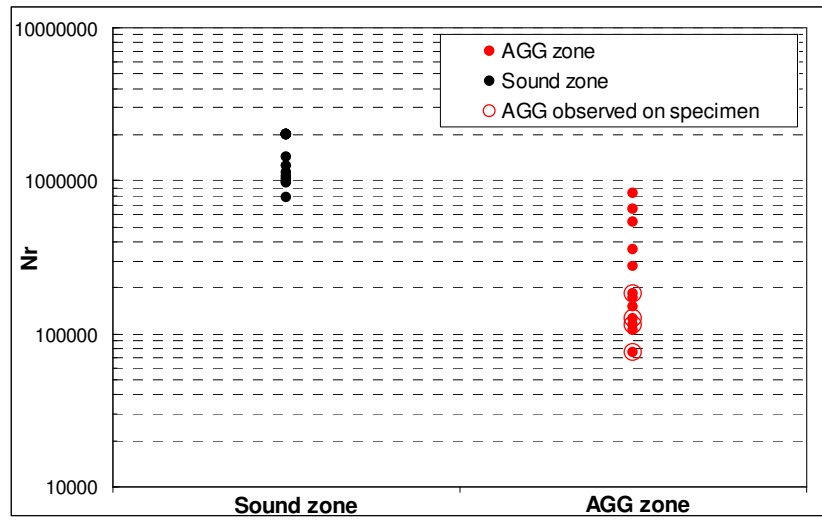

(a)

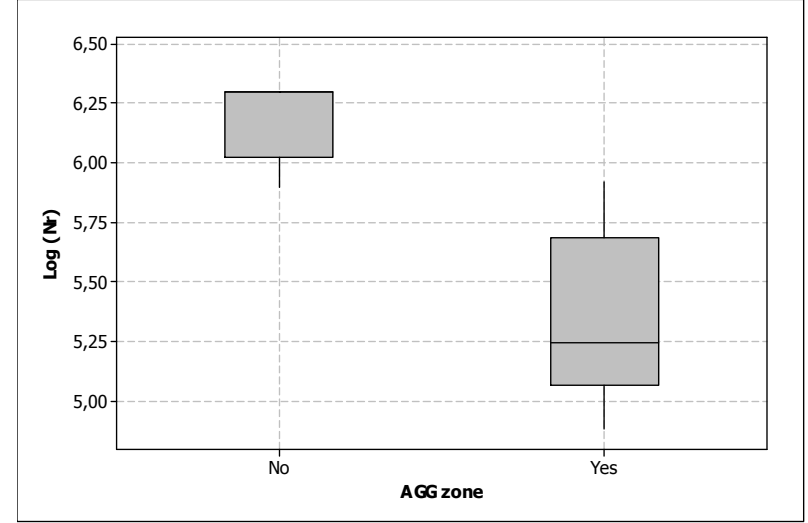

(b)

Figure 6: Fatigue results

After testing, failed specimens were all examined by means of SEM in order to identify the main crack initiation site. Figure 7a shows a fractography representative of the specimens cut in AGG zones. The crack initiation site can clearly be identified on the specimen's surface on a single sheared coarse grain exhibiting either a single facet or a chevron facet. In all cases, the grain identified to originate crack initiation area has been measured and is consistent with the size of the coarsest grains observed with EBSD on the specimen of zone 2, i.e. about 70 to $100 \mu \mathrm{m}$.

Large grains lead to larger stress concentration at the grain boundaries and early crack initiation due to long slip lengths promoting cyclic strain localization. It is important to note that in most cases, coarse grains that are identified in the crack initiation area are totally cut, from one side to another. In some instances, and as illustrated in Figure 7d, the grain can exhibit chevron facet. Such single plane or chevron facet initiation indicate initiation along twin boundaries and has already been reported on Waspaloy LCF specimens tested at $538^{\circ} \mathrm{C}$ [8] or in René 88DT tested in ultrasonic fatigue at $593^{\circ} \mathrm{C}$. [9]. Miao et al. [9] as well as Llanes and Laird [10] tend to confirm that twin boundaries are preferred sites for early formation of persistent slip bands. This is even more the case nearby discontinuities such as twin steps.

A longitudinal cross section of all the broken LCF samples has also been observed by means of optical microscopy in order to confirm the presence of AGG zones. All the specimens in which some coarse grain areas have been identified have been circled in red in Figure 6a. It appears that all the lowest points correspond to the specimens containing AGG. Figure 8 shows a picture of the typical microstructure obtained on AGG containing specimens. 


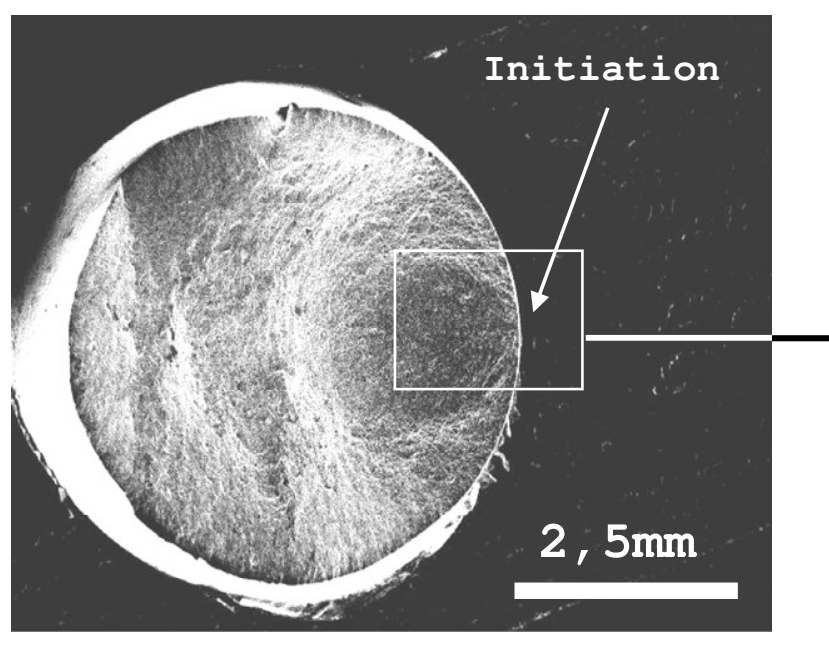

(a)

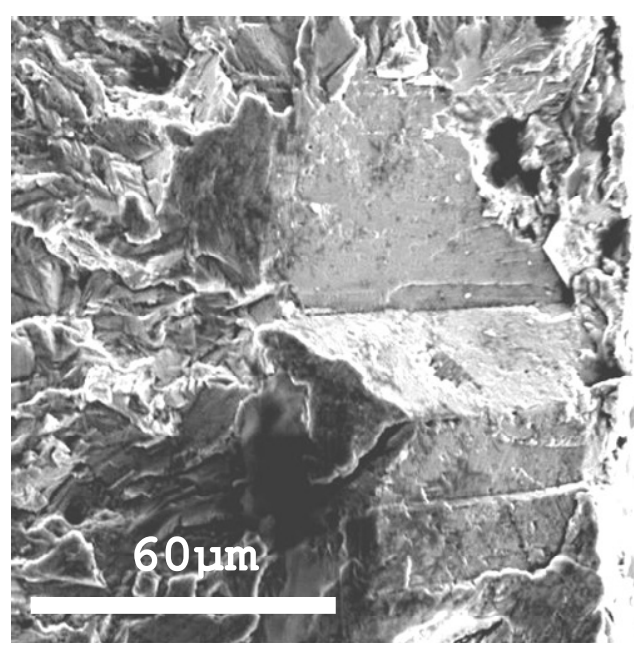

(d)

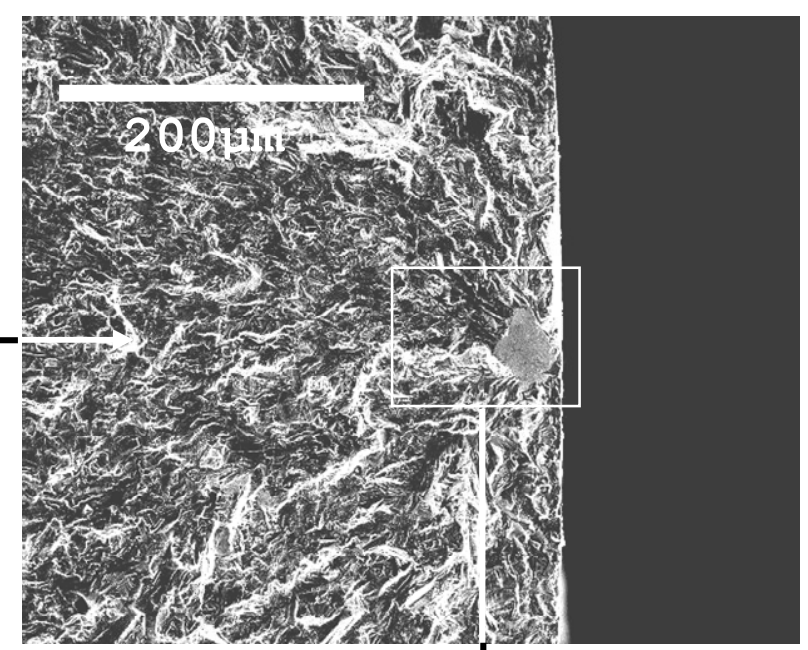

(b)

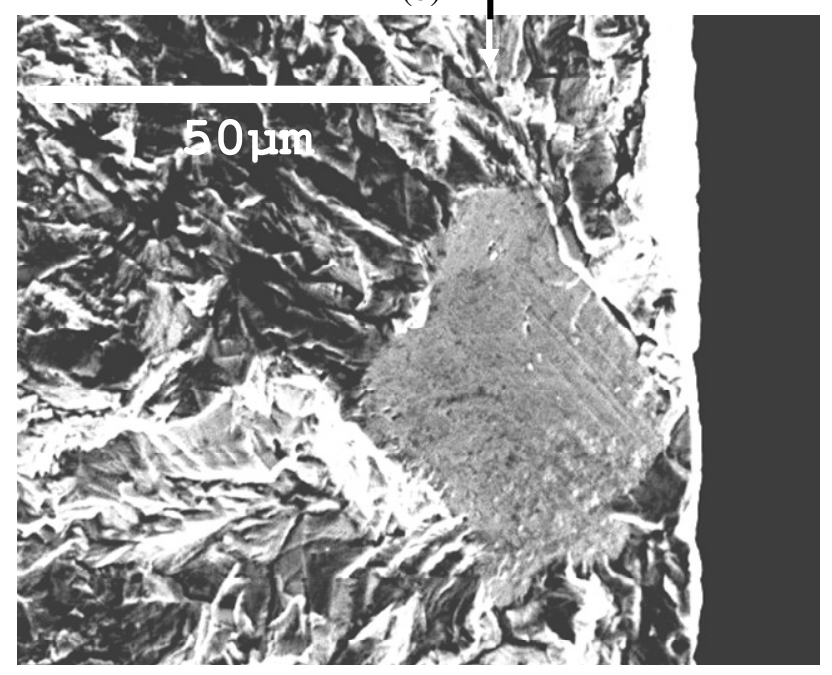

(c)

Figure 7: Fractography of a broken LCF specimen cut in AGG zone.

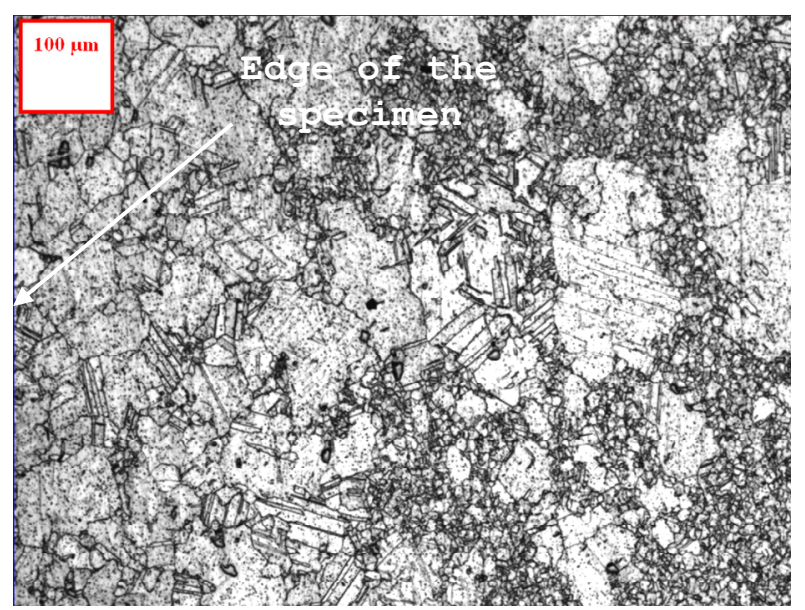

(a)

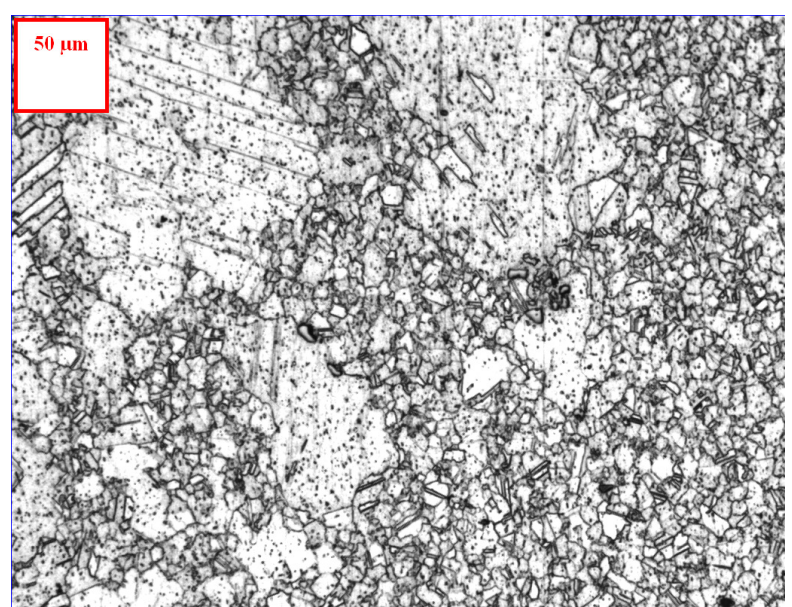

(b)

Figure 8: Microstructure of an AGG-containing specimen 
These results should be compared with those obtained on specimens that do not contain AGG microstructures. In such homogeneous microstructures, the main crack very commonly initiates on a crystallographic cleavage on a grain located on the surface of the specimen [13]. The crack initiation mode is thus very similar to what has been identified on the AGG containing samples. In some instances however, crack initiation can occur on carbonitride particles formed during solidification and which are located at the surface or close to the surface of the specimen. This type of initiation generally leads to lower fatigue results and the scatter observed in the fatigue results of fine grained Alloy 718 . This can be explained by high strain incompatibilities at the carbide/matrix interface particles that can lead to decohesion phenomena and rapid crack initiation during the first cycles [12]. This type of fracture surface is illustrated in Fig. 9.

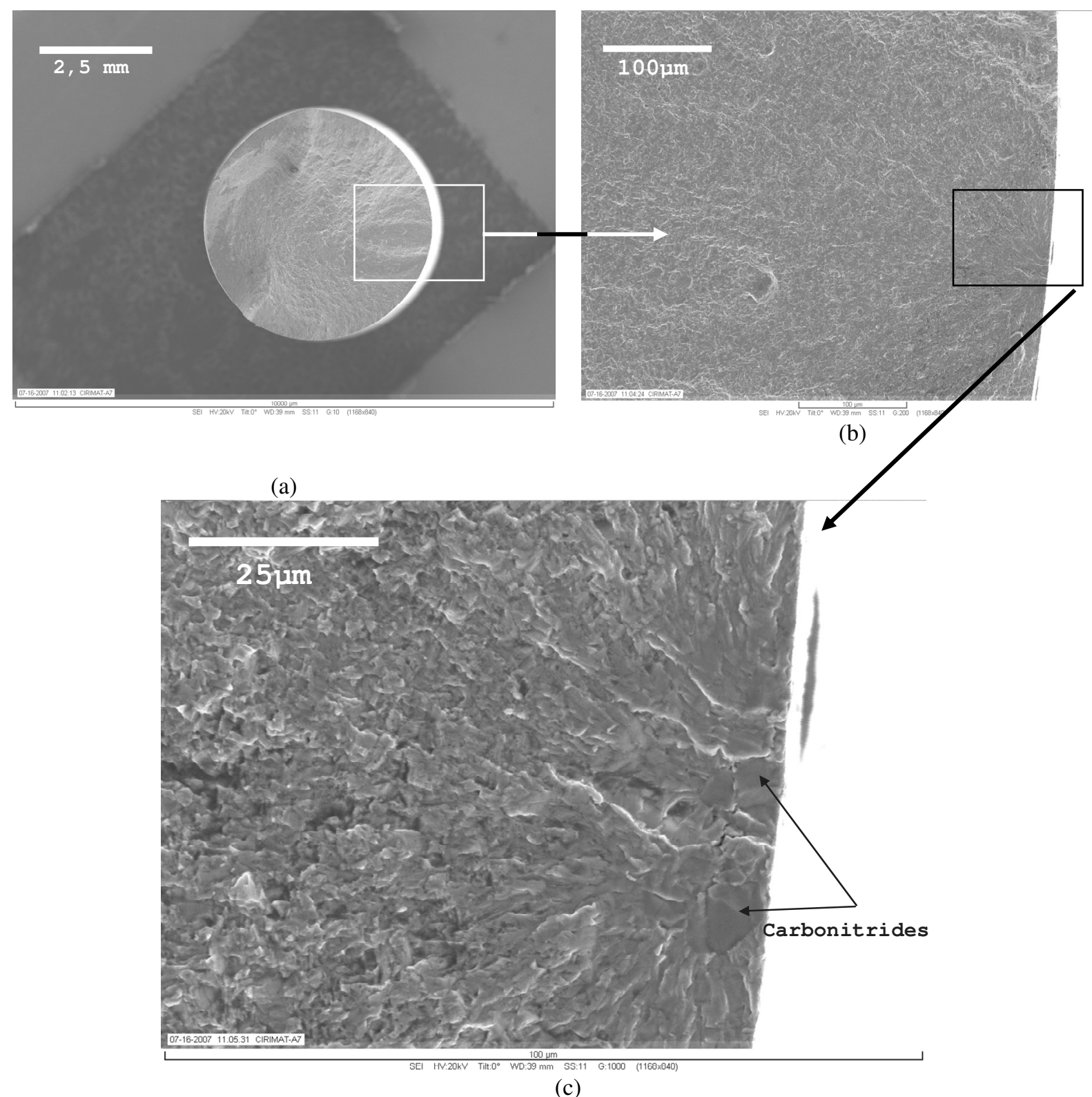

Figure 9: Fractography of a broken specimen free of AGG exhibiting surface crack initiation on carbonitride particles

The results exposed above show that the presence of AGG zones in the LCF specimens lead to a strong decrease of the fatigue lifetime even if the main crack initiates on a 
crystallographic cleavage in both cases. The presence of larger grains in AGG containing specimens may promote larger strain concentration at the grain boundaries due to longer slip lengths and larger dislocation pile-ups. Considering that the fatigue lifetime can be divided into a step 1 crack initiation process on the largest grains followed by a step 2 crack propagation step, we can assess the number of cycles to failure of the specimens. The number of cycles necessary to initiate a crack can be calculated based on the plastic strain amplitude using Tanaka and Mura [14] model (see Equation 1). The number of cycles necessary to propagate the crack to the fracture of the specimen can be calculated by integrating a Paris law (see Equation 2) and considering that the average grain size of the specimen remains the same on the specimens with and without AGG. The parameters $\mathrm{C}$ and $\mathrm{n}$ are thus the same and have been determined experimentally at $350^{\circ} \mathrm{C}$ and are presented in Table 2 . The final crack size has been chosen as $3 \mathrm{~mm}$, but its actual value does not influence the result much, as far as the number of cycles necessary for the crack propagation is much larger for short crack lengths.

$$
\begin{aligned}
& N i=\alpha \frac{1}{d \times \Delta \varepsilon_{p}^{2}} \\
& \frac{d a}{d N}=C \Delta K^{n}
\end{aligned}
$$

The Stress intensity factor has been calculated using equation 3 considering a constant stabilized stress of 1000MPa and Foreman and Shivakumar formulation [11] assuming that the crack propagates as a circular arc in the cylindrical specimen.

$$
\begin{gathered}
K=\sigma_{0} F_{0}(\lambda) \sqrt{\pi a} \\
\text { where } \\
F=g(\lambda)\left[0,752+2,02 \lambda+0,37\left(\sin \frac{\pi \lambda}{2}\right)^{3}\right] \\
\text { and } \lambda=\frac{a}{d}
\end{gathered}
$$

Table 2: Crack propagation experimental data used for calculations.

Sinusoidal waveform $\mathrm{R}=0,05,350^{\circ} \mathrm{C}, 2 \mathrm{~Hz}$

\begin{tabular}{|c|c|}
\hline $\mathbf{C}(\mathbf{m} /$ cycle $)$ & $\mathbf{n}$ \\
\hline $6,22 \mathrm{E}-12$ & 3,01 \\
\hline
\end{tabular}

The number of cycles to fracture has been calculated as a function of the size of the coarsest grain on which initiation occurs, which spans between a few microns for the specimens not containing AGG to several tens of microns in the case of AGG containing specimens. The calculated number of cycles for step 1 initiation, for propagation and the calculated total number of cycles are compared to the experimental results (see Fig. 10). 
These results show that the presence of islands of coarse grains are responsible for early crack initiation and that the subsequent total number of cycles to failure can be described using a simple approach based on the size of the coarsest grain present in the material and Tanaka and Mura model for the crack initiation process.

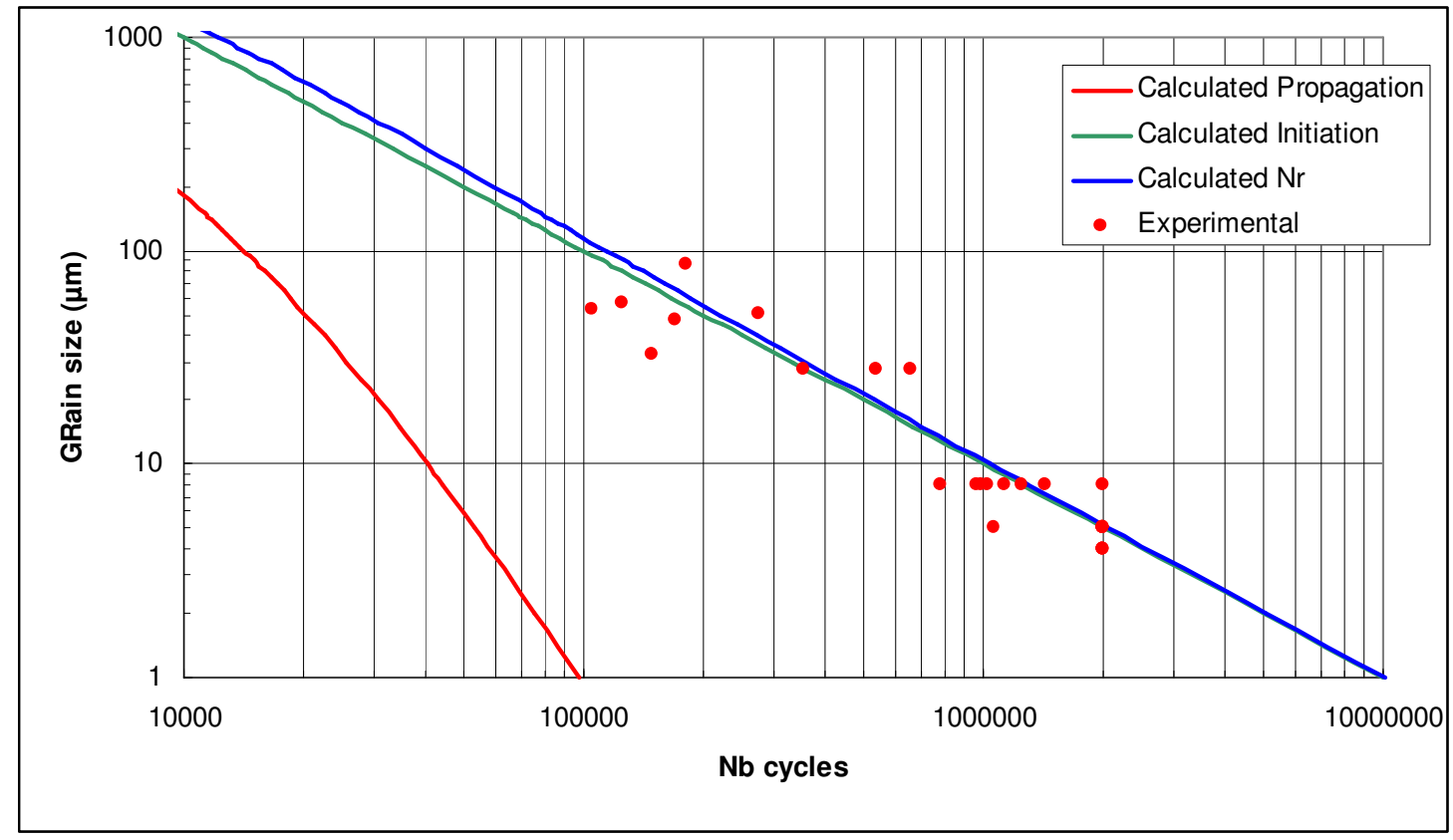

Figure 10: Calculated number of cycles to failure. Comparison with experimental results.

\section{Concluding remarks}

Abnormal grain growth zones of a forging have been characterized both on a microstructural and a mechanical point of view. The microstructure consists in a very fine grained matrix containing some very large grains up to $100 \mu \mathrm{m}$. The coarse grain areas do not seem solute lean and the amount of the grain size controlling delta phase is similar as in fine grain zones. Although some additional investigations are needed at a finer scale, no particular local texture has been identified as a possible cause of the abnormal growth phenomenon.

The consequences of the presence of $\mathrm{AGG}$ zones on $350^{\circ} \mathrm{C}$ tensile properties seem very limited, but they are very significant on the fatigue lifetime. The lifetime decrease in the conditions chosen here can reach one decade and is associated to early crack initiation on the coarsest grains located at the specimen's surface intersection. The lifetime can be successfully described based on Tanaka and Mura model for crack initiation and considering the size of the coarsest grain present on the fracture surface.

In addition to the necessity to investigate the mechanism that leads to the presence of local AGG zones in a forged part, this work points out the importance of coarse grain islands on the fatigue durability of alloy 718 .

\section{References}

1. N. Maazi et al., "Influence of neighbourhood on abnormal Goss grain growth in $\mathrm{Fe}-3 \% \mathrm{Si}$ steels: Formation of island grains in the large growing grain", Scripta Materialia, 55 (2006), 641644. 
2. Hyun-Chul Kim et al., "Effect of primary recrystallization texture on abnormal grain growth in an aluminum alloy", Scripta Materialia, 57 (2007), 325-327.

3. A.L. Etter, T. Baudin and R. Penelle, "Influence of grain environment during secondary recrystallization of conventional grain oriented Fe-3\% Si steels", Scripta Materialia, 47 (2002), 725-730.

4. P.R. Rios, "Abnormal Grain Growth Development from Uniform Grain Size Distributions due to a Mobility Advantage", Scripta Materialia, 38 (12) (1998), 1359-1364.

5. F.J. Humphreys, "A unified theory of recovery, recrystallization and grain growth, based on the stability and growth of cellular microstructures-I. The basic model", Acta Materialia, 45 (10) (1997), 4231-4240.

6. F.J. Humphreys, "A unified theory of recovery, recrystallization and grain growth, based on the stability and growth of cellular microstructures-II. The effect of second-phase particles", Acta Materialia, 45 (12) (1997), 5031-5039.

7. M. Hillert, "On the theory of normal and abnormal grain growth", Acta Metallurgica, 13 (3) (1965), 227-238.

8. M.L. Brogdon and A.H. Rosenberger, "Evaluation of the influence of grain structure on the fatigue variability of Waspaloy", (Paper presented at Superalloys 2008, Champion, PA, USA, 2000), 583-588.

9. J. Miao, T.M. Pollock and J.W. Jones, "Fatigue crack initiation in nickel based superalloy René 88 DT at $593^{\circ} \mathrm{C} "$, (Paper presented at Superalloys 2008, Champion, PA, USA, 2000), 589597.

10. L. Llanes and C. Laird,"The role of annealing twin boundaries in the cyclic deformation of f.c.c. materials", Materials Science and Engineering A, 157 (1) (1992), 21-27.

11. R.G. Forman and V. Shivakumar, "Growth behavior of surface cracks in circumferential plane of solids and hollow cylinders", Fracture Mechanics, 17 (1986), 59-74.

12. F. Alexandre, S. Deyber and A. Pineau, "Modelling the optimum grain size on the low cycle fatigue life of a Ni based superalloy in the presence of two possible crack initiation sites", Scripta Materialia, 50 (2004), 25-30.

13. N. Späth et al., "718 superalloy forging simulation: a way to improve process and material potentialities", (Paper presented at Superalloys 718, 625, 706 and Various Derivatives, 2001), 173-183.

14. K. Tanaka and T. Mura, "A dislocation model for fatigue crack initiation", Journal of Applied Mechanics, 48 (1981), 97-103. 